\title{
Volumetric Measurement of Ground-Glass Opacity Nodules using Expectation-Maximization Algorithm
}

\author{
Yanli Miao a, Jianming Wang a , Weiwei Du, ${ }^{\mathrm{b},}$, Yanhe $\mathrm{Ma}^{\mathrm{c}}$, Hong Zhang ${ }^{\mathrm{c}}$ \\ aTianjin Polytechnic University, Xiqing District, Tianjin 300387, China \\ ${ }^{b}$ Kyoto Insititute of Technology, Sakyoku, Kyoto 6068585, Japan \\ 'Tianjin Chest Hospital, Jinnan District, Tianjin 300051, China \\ *Corresponding Author: duweiwei@kit.ac.jp
}

\begin{abstract}
This paper proposes Expectation-Maximization (EM) algorithm to segment pure Ground-Glass Opacity (GGO) nodules and solid part in part-solid GGO nodules and obtain their volumes as the features of GGO nodules. The features of GGO nodules are helpful to determine whether the GGO nodule is malignant. EM algorithm is used to segment pure GGO nodules and solid part in part-solid GGO nodules with different parameters. Experiments show EM algorithm can quickly segment solid part in part-solid GGO nodules and pure GGO nodules to reduce the burden of doctors.

Keywords: Volumetric measurement, Solid part in part-solid Ground-Glass Opacity nodules, No solid part in part-solid Ground-Glass Opacity nodules, pure Ground-Glass Opacity nodules, Expectation-Maximization algorithm.
\end{abstract}

\section{Introduction}

Lung cancer has a higher mortality rate than others in World Health Organization ${ }^{(1)}$. One of reasons is that the tumor has been a malignant tumor when it was found from health diagnosis. Survival rate of lung cancer is less than $16 \%$ according to the survey in 5 years. Survival rate of lung cancer becomes $90 \%$ in 5 years if tumors of lung have been found early. When tumors process to the stages of malignant, survival rate of lung cancer in I stage is $60 \%$, survival rate of lung cancer reduce $5 \%$ of IV stage from $40 \%$ of II stage. Therefore it is important to detect tumors of lung at earlier stages ${ }^{(2)}$.

The target of this paper is Computed Tomography (CT) images which are one of image diagnostics to detect malignant pulmonary nodules. The pulmonary nodules are detected with some features of pulmonary nodules such as the size, morphological characteristics and position of lungs in image diagnostics ${ }^{(3)}$. Thus, these researches on detection of malignant pulmonary nodules were proposed (4), especially, segmentation and the features extraction of solid pulmonary nodules using image processing methods ${ }^{(5),(7)}$.

Pulmonary nodules have two types: Solid nodules and Ground-Class Opacity (GGO) nodules. The GGO nodules maybe malignant if there is solid part in GGO nodules called part-solid GGO nodules ${ }^{(6)}$. However, there are low contrast between GGO nodules and tissue surrounding GGO nodules. It is a tough task to obtain segmentation or some features extraction of GGO nodules.

This paper proposes Expectation-Maximization (EM) algorithm to segment pure Ground-Glass Opacity (GGO) nodules and solid part in part-solid GGO nodules and obtain their volumes as the features of GGO nodules. The features of GGO nodules are helpful to determine whether the GGO nodule is malignant. EM algorithm is used to segment pure GGO nodules and solid part in part-solid GGO nodules with different parameters. Experiments show EM algorithm can quickly segment solid part in part-solid GGO nodules and pure GGO nodules to reduce the burden of doctors.

The remainder of this is organized as follows. Section II introduces our proposal. Section III gives some experiments to measure the volumes of GGO nodules and solid part in GGO nodules. Section VI draws conclusions and future work.

\section{Methodology}

There are two types on GGO nodules. One is that there is solid part in GGO nodules called part-solid GGO nodules, and the other is that there is no solid part in GGO nodules called pure GGO nodules. The part-solid GGO nodules 
include solid part and no solid part. As the GGO nodules are maybe malignant if there is solid part in GGO nodules, segmentation or some features extraction of GGO nodules are important to image diagnostics. This study proposes EM algorithm to segment pure GGO nodules and solid part in part-solid GGO nodules.

EM algorithm ${ }^{(8)}$ is an unsupervised learning to find latent information based on iteration. There are two clusters to part-solid GGO nodules. One cluster is solid part in partsolid GGO nodules, and the other is no solid part in part-solid GGO nodules. The two clusters are considered as latent information of EM algorithm. Suppose that the two clusters are Gaussian distribution.

Volumes of GGO nodules can be measured by the flowchart which showed at Fig. 1.

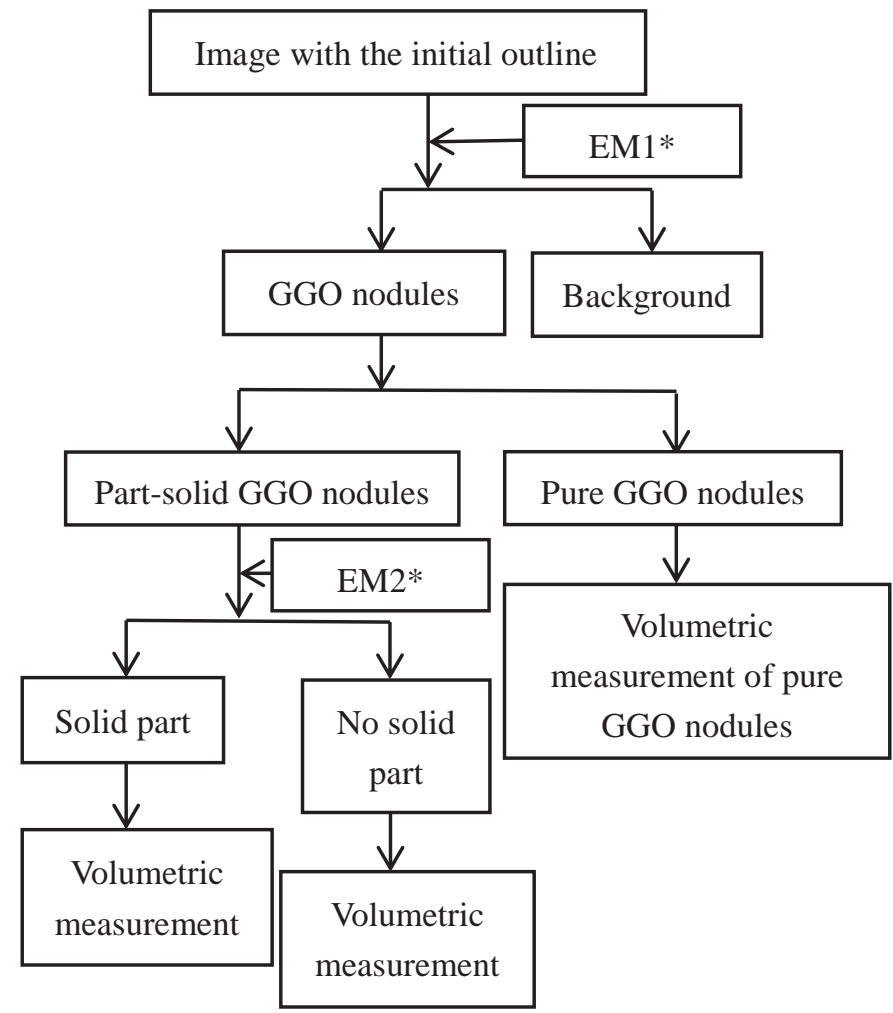

Fig.1. A flowchart of volumetric measurement of GGO nodules, *EM1 and EM2 have different parameters.

First, the initial outlines were drawn to GGO nodules in slices in CT volume data. GGO nodules were segmented from the images using EM algorithm. The pure GGO nodules can be segmented directly and obtained its volume. The part-solid GGO nodules need to use EM algorithm again to segment solid part and no solid part in part-solid GGO nodules, and then their volumes can be obtained. Note that the parameters on twice of EM algorithm are different.
This paper proposes EM algorithm in order to find the clusters of GGO nodules. The clusters can be obtained as the followings.

(1). To initialize the mean and variance of pixels value from the image with the initial outline.

(2). To compute the expectation based on the mean and variance of (1). It is called E step.

(3). To compute the new mean and variance based on the expectation (2). It is called M step.

(4). To check the convergence of the mean and variance. If the convergence criterion is not satisfied, the computation returns to (2).

\section{Experimental results}

The data are provided by Tianjin chest hospital, China and from Philips muiltiCT for scanning: the scanning range is from the thoracic inlet to the lung bottom. 5 GGO nodules were experimented. The age range of the patients with 5 GGO nodules is from 57 to 78 years old. Some parameters of CT volume data are showed as follows:

Tube voltage is $120 \mathrm{KV}$ and tube current is $250 \sim 450 \mathrm{~mA}$. The collimator width is $128 \times 0.6 \mathrm{~mm}$ and the Rpm of bulb is $0.27 \mathrm{~s} /$ round; the reconstruction slice thickness is $1.5 \mathrm{~mm}$. The layer interval is $0.5 \mathrm{~mm}$ and the resolution of one slice is $512 \times 512$ pixels.

3 methods are given in section 3.1, 3.2 and 3.3 and explained by the example of a part-solid GGO nodule. This study proposes section 3.2. Section 3.3 is a simple threshold method. Section 3.4 gives other examples including pure GGO nodules and part-solid GGO nodules.

The same initial outline was given to the part-solid GGO nodules. The number of clusters and iteration are parameters of EM algorithm in section 3.1 and 3.2. Suppose that one cluster is one Gaussian distribution model.

\subsection{EM algorithm with the same parameters}

Number of the clusters is 8 and iteration is 3 by experience in EM algorithm with the same parameters. EM1 and EM2 of Fig. 1 mean that there are the same parameters. The results were shown at EM with the same parameters column of table 1. Blank means that solid part in part-solid GGO nodules cannot be segmented or there were no solid part from slice 82 to 86 and 95 to 96 . 


\subsection{EM algorithm with the different parameters}

Number of the clusters is 8 and iteration is 2 in EM1 of Fig. 1. Number of the clusters is 20 and iteration is 4 in EM2 of Fig. 1. The parameters are determined by experience. The results were shown at EM with the different parameters column of table 1. Blank means that solid part in part-solid GGO nodules cannot be segmented or there were no solid part from slice 82 to 86 and 95 to 96 .

\subsection{A threshold method}

Two threshold values had been set. One is 75 to segment part-solid GGO nodules or pure GGO nodules, and the other is 150 to segment solid part in part-solid GGO nodules. Histogram of table 1 means that the image of the initial outline. The two thresholds values were difficult to be determined from histogram column of table 1. Blank means that solid part in part-solid GGO nodules cannot be segmented or there were no solid part from slice 82 .

\subsection{Volumetric measurement of part-solid GGO nodules and pure GGO nodules}

5 GGO nodules were experimented to measure their volumes. GGO1, GGO2, GGO3 and GGO5 are part-solid GGO nodules. GGO4 is a pure GGO nodule. There are two values to show volumes to part-solid GGO nodules. The upper value is the volume of whole part-solid GGO nodules. The lower value is the volume of solid part in part-solid GGO nodules. EM(3.1) represents the EM algorithm with the same parameters. EM(3.2) represents the EM algorithm with the different parameters. Threshold represents the threshold method. The volume of GGO nodules can be computed by the following formula. $\mathrm{N}$ is the number of slices including the GGO nodule.

Volume of a GGO=

$$
\sum_{i=1}^{N} \text { (number of pixels of } G G O \times \text { size of a pixel }{ }^{2}
$$

$\times$ thichkness of a slice)

Additionally, Philips companion provides the software to segment GGO nodules which were showed in Fig. 2. The software only views GGO nodules and cannot compute the volume of solid part in part-solid GGO nodules.
Table 2. Volumetric measurement GGO nodules based on section 3.1, 3.2 and 3.3.

\begin{tabular}{|c|c|c|c|}
\hline $\begin{array}{l}\text { Number of } \\
\text { GGO } \\
\text { nodules }\end{array}$ & $\operatorname{EM}(3.1)$ & $\operatorname{EM}(3.2)$ & Threshold \\
\hline \multirow{2}{*}{$\begin{array}{l}\text { GGO1 } \\
\left(\mathrm{mm}^{3}\right)\end{array}$} & 8516.4 & 8013.0 & 7421.3 \\
\hline & 3684.6 & 344.8 & 2147.0 \\
\hline \multirow{2}{*}{$\begin{array}{l}\text { GGO2 } \\
\left(\mathrm{mm}^{3}\right)\end{array}$} & 4857.7 & 4857.7 & 4877.6 \\
\hline & 2072.7 & 3722.1 & 4371.0 \\
\hline \multirow{2}{*}{$\begin{array}{l}\text { GGO3 } \\
\left(\mathrm{mm}^{3}\right)\end{array}$} & 10280.0 & 9362.7 & 9197.6 \\
\hline & 7086.4 & 5381.2 & 2229.6 \\
\hline $\begin{array}{l}\text { GGO4 } \\
\left(\mathrm{mm}^{3}\right)\end{array}$ & 2669.3 & & 1198.2 \\
\hline \multirow{2}{*}{$\begin{array}{l}\text { GGO5 } \\
\left(\mathrm{mm}^{3}\right)\end{array}$} & 3531.1 & 2946.2 & 2995.6 \\
\hline & 2533.7 & 821.9 & 1112.3 \\
\hline
\end{tabular}

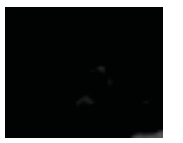

87

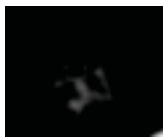

91

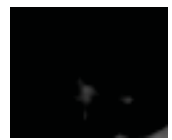

88

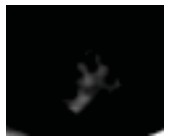

92

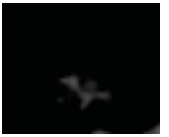

89

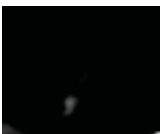

93

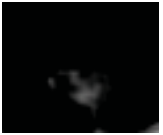

90

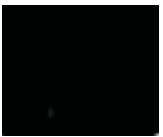

94
Fig. 2. A GGO nodule was segmented using the software provided by Philips companion

\section{Conclusions}

EM algorithm has been proposed to segment GGO nodules and obtain their volumes. Experiments show EM algorithm can quickly segment solid part in part-solid GGO nodules and pure GGO nodules to reduce the burden of doctors. To increase the number of GGO nodules to confirm the effectiveness of our approach is our further work. 
Table 1. Experimental results on section 3.1, section 3.2 and section 3.3

\begin{tabular}{|c|c|c|c|c|c|c|c|c|}
\hline $\begin{array}{l}\text { No. } \\
\text { in }\end{array}$ & $\begin{array}{l}\text { Images } \\
\text { with initial }\end{array}$ & $\begin{array}{l}\text { EM wit } \\
\text { parameters }\end{array}$ & the same & $\begin{array}{l}\text { EM with } \\
\text { parameters }\end{array}$ & he different & Histogram & A threshol & ethod \\
\hline $\begin{array}{l}\text { slice } \\
\text { s }\end{array}$ & outline & Solid part & $\begin{array}{l}\text { GGO } \\
\text { nodules }\end{array}$ & Solid part & $\begin{array}{l}\text { GGO } \\
\text { nodules }\end{array}$ & & Solid part & $\begin{array}{l}\text { GGO } \\
\text { nodules }\end{array}$ \\
\hline 82 & & & & & & & & \\
\hline 83 & & & & & & & & \\
\hline 84 & & & & & & & & \\
\hline 85 & & & & & & & & \\
\hline 86 & & & & & & & & \\
\hline 87 & & & & & & & & \\
\hline 88 & & & & & & & & \\
\hline 89 & & & & & & & & \\
\hline 90 & & & & & & & & \\
\hline 91 & & & & & & & & \\
\hline 92 & & & & & & & & \\
\hline 93 & & & & & & & & \\
\hline 94 & & & & $=$ & & & 1 & \\
\hline 95 & & & & & & & & \\
\hline 96 & & & & & & & & \\
\hline
\end{tabular}




\section{References}

(1) R. Siegel, D. Naishadham, and A. Jemal, "Cancer Statistics," CA Cancer J. Clin., vol. 63, pp. 11-30, 2013.

(2) C. I. Henschke, D. I. McCauley, D. F. Yankelevitz, D. P. Naidich, G. McGuinness, O. S. Miettinen, D. M. Libby, M. W. Pasmantier, J. Koizumi, N. K. Altorki, and J. P. Smith, "Early Lung Cancer Action Project: Overall Design and Findings from Baseline Screening," Lancet, vol. 354, no. 9173, pp. 99-105, 1999.

(3) H. MacMahon, J. H. M. Austin, G. Gamsu, C. J. Herold, J. R. Jett, D. P. Naidich, E. F. Patz, and S. J. Swensen, "Guidelines for Management of Small Pulmonary Nodules Detected on CT scans: A Statement from the Fleischner Society," Radiology, vol. 237, no. 2, pp. 395400, 2005

(4) H. Han, L. Li and F. Han : "Fast and Adaptive Detection of Pulmonary Nodules in Thoracic CT Images Using a Hierarchical Vector Quantization Scheme", IEEE Journal of Biomedical and Health Informatics, Vol, 19, No. 2, pp. 648-659, 2015

(5) O. Elsayed, K. Mahar and M. Kholief : "Automatic Detection of the Pulmonary Nodules from CT Images", SAI Intelligent Systems Conference, No. 10-11, pp. 742-746, 2015

(6) H. Chae, C. Park and S. Park: "Computerized Texture Analysis of Persistert Part-Solid Ground-Glass Nodules : Differentiation of Preinvasive Lesions from Invasive Pulmonary Adenocarcinomas", Radiology, Vol 273, No. 1, pp. 285-293, 2014

(7) A. Yaguchi, T. Okazakia and T. Takeguchi, "SemiAutomated Segmentation of Solid and GGO Nodules in Lung CT Images using Vessel-Likelihood Derived from Local Foreground Structure", Proc. Of SPIE, Vol 9414, pp. 742-746, 2015

(8) G.M. McLachlan and T. Krishnan: "The EM Algorithm and Extensions", Journal of Classification, Vol, 15, pp. 154-156, 1988

(9) DICOM Conformance Statement for DICOM Viewer Release 3.0, Philips Healthcare, 2013 